\title{
Phase transitions during membrane formation of polylactides. I. A morphological study of membranes obtained from the system polylactide-chloroform-methanol
}

\author{
P. van de Witte, H. Esselbrugge, P.J. Dijkstra, J.W.A. van den Berg, J. Feijen * \\ Department of Chemical Technology, Biomaterials Section and Institute for Biomedical Technology (BMTI), University of Twente, \\ P.O. Box 217, 7500 AE Enschede, Netherlands
}

Accepted 27 February 1995

\begin{abstract}
The influence of solid-liquid demixing, liquid-liquid demixing and vitrification on the morphology of polylactide membranes has been investigated. To study the effects of crystallization of polylactides on the membrane formation and morphology, polylactides of varying stereoregularity were used. The polymers applied were poly-L-lactide (PLLA) and copolymers with different molar ratios of L-lactide and D-lactide [poly-L95/D5-lactide (PLA95), poly-L80/D20-lactide (PLA80) and polyL50/D50-lactide (PDLLA) ]. Solutions of polylactides in chloroform cast on a glass plate were immersed in methanol. From solutions containing the slowly crystallizing PLA80 or uncrystallizable PDLLA porous membranes were obtained if the phase separated system was removed from the nonsolvent bath within a few hours after immersion. After longer equilibration times in methanol the structure collapsed. The swelling in the nonsolvent methanol was too high to allow stabilization of the liquidliquid demixed structure by vitrification. Stable membranes were easily obtained with more rapidly crystallizing polymers like PLLA. Casting solutions with low PLLA concentrations gave membranes with a cellular morphology due to liquid-liquid demixing by nucleation and growth of a polymer poor phase. Crystallization only played a role in the fixation of the liquidliquid demixed structure. At increasing PLLA concentrations the demixing sequence gradually reversed to crystallization followed by liquid-liquid demixing. In these cases membranes with porous spherulites or spherulites surrounded with a cellular layer were obtained.
\end{abstract}

Keywords: Polylactides; Morphology; Liquid-liquid demixing; Crystallization

\section{Introduction}

Porous membranes can be prepared using a variety of techniques [1]. Many of the commercially available membranes are prepared using the immersion precipitation process. The principle steps of this method involve casting of a polymer solution onto an inert support followed by immersion of the support with the

\footnotetext{
* Corresponding author.
}

cast film into a bath filled with a nonsolvent for the polymer. The subsequent exchange of solvent for nonsolvent induces phase separation of the polymer solution. The phase separation process responsible for the porous cellular structure of most membranes is liquidliquid demixing by nucleation and growth of a polymer poor phase. In principle liquid-liquid phase separation can proceed until two fully separated layers are obtained. To obtain membranes with a stable porous morphology the liquid-liquid phase separation process 
has to be arrested before completion. The demixed solution is solidified when a gel transition is passed in the course of the demixing process. Several physical phenomena can induce the formation of a gel [2]. Examples are gelation by association of components in the solution, gelation by vitrification or gelation by crystallization. During the last few years the implications of combinations of phase transitions for the membrane morphology have received considerable attention [3-6]. However the studies are usually limited to thermally induced phase separation of binary polymer solutions.

The number of studies in which the relation between gel transitions and experimental membrane morphologies of membranes obtained by immersion precipitation is explicitly discussed is still low (see for example $[1,7-11]$ ). Usually the attention is focused on liquidliquid demixing. Recently the role of solid-liquid demixing processes on the membrane morphology has been addressed by Bulte et al. for the system nylon4,6-formic acid-water [12]. The structure formation of this system proceeded almost entirely via solid-liquid demixing and resulted in the formation of membranes with spherulitic and axialitic structures. Cellular membranes were only obtained with casting solutions with a very low polymer concentration.

The importance of solid-liquid demixing processes during immersion precipitation was also demonstrated for the wet-spinning of poly-L-lactide (PLLA) hollow fibres [13-15]. Depending on the solvent-nonsolvent combination used, fibres could be obtained with either cellular morphologies in the sublayer (structure largely due to liquid-liquid demixing) or with a leafy morphology (structure due to solid-liquid demixing). In particular fibres with a morphology induced by solidliquid demixing processes were useful for the controlled delivery of drugs.

The purpose of the present study is to study the influence of liquid-liquid demixing, solid-liquid demixing and vitrification on the morphologies of polylactide membranes obtained via immersion precipitation. It is attempted to relate the morphologies to the phase diagrams of the ternary systems. Several stereoisomers of polylactides have been used. The ability of the polymer to crystallize can easily be varied without strongly varying the chemical composition of the polymers. The other physical properties of the polymers are expected to be similar. This feature makes it pos- sible to distinguish between effects due to solid-liquid demixing and effects due to other transitions. Amorphous polymers can be obtained by random copolymerization of a racemic mixture of L-lactide and D-lactide. An increase of the stereoregularity of the polymer results in a strong increase of the crystallization rate [16]. In this study PLLA (100\% L-units), PLA95 (95\% L-units, 5\% D-units) and PLA80 (80\% L-units, $20 \%$ D-units) were used. Chloroform was used as a solvent and methanol as a nonsolvent. Isothermal ternary phase diagrams for the highly crystalline PLLA and the amorphous PDLLA have been constructed. Membranes of all polylactides mentioned above were prepared.

\section{Experimental}

\subsection{Synthesis and characterization of the polymers}

PLLA was synthesized from L-lactide (Purac Biochem). PDLLA was synthesized from a racemic mixture of L-lactide and D-lactide. The other copolymers are referred to as PLA80 and PLA95, the numbers between the brackets indicate the percentage of L-lactide used in the feed. Each monomer was recrystallized from sodium distilled toluene. The monomers were mixed thoroughly in the polymerization tubes. Polymerizations were carried out in the melt $\left(130^{\circ} \mathrm{C}\right)$ in vacuo with stannous-octoate (Sigma chemicals) as catalyst [17]. The polymers were purified by dissolving the polymer in chloroform and precipitating the polymer in methanol. Afterwards the polymers were dried for at least $48 \mathrm{~h}$ in vacuo at $85^{\circ} \mathrm{C}$. DSC scans were performed on a Perkin-Elmer DSC7. The polymer samples were slowly cooled from the melt to $60^{\circ} \mathrm{C}\left(2^{\circ} \mathrm{C} /\right.$ min) and reheated after $30 \mathrm{~min}$ at a scan rate of $10^{\circ} \mathrm{C} /$ $\min$. The end point of the endotherm was taken as the melting point. Intrinsic viscosities of polymers in chloroform $\left(25^{\circ} \mathrm{C}\right)$ were determined with an Ubbelohde viscometer using the standard extrapolation of the reduced viscosity to zero concentration. The optical rotation, $[\alpha]_{\mathrm{D}}^{25}$, was determined using polymer solutions in dichloromethane $(1 \% \mathrm{w} / \mathrm{v})$ and a PerkinElmer 241 polarimeter. The optical purity of the polymers was calculated using the value for $100 \%$ optically pure poly-L-lactide as given by Kricheldorff [18]. 


\subsection{Casting solutions}

Chloroform was obtained from Merck and was of analytical quality. Polymer solutions were prepared by slowly stirring the components for 2 days. The solution containing $30 \% \mathrm{w} / \mathrm{w}$ PLLA was only stable for a limited time. Within $24 \mathrm{~h}$ after preparation of the solution a small amount of crystallites precipitated. Therefore this solution was prepared at elevated temperature and used directly after cooling to room temperature. All other solutions were stable for a much longer period of time.

\subsection{Membranes}

Membranes were prepared by casting the polymer solution on a glass plate with a doctor's blade and immersing the solution in methanol (technical quality, purity as indicated by gas chromatography $>99 \%$ ). The temperature of the bath was kept at $22 \pm 2^{\circ} \mathrm{C}$. The initial casting thickness was $400 \mu \mathrm{m}$. The time between casting and immersion amounted to circa $10 \mathrm{~s}$. The membranes were kept in the nonsolvent for a period of 3 days and dried afterwards ( 1 day air drying, 1 day vacuum). Thereafter the membranes were characterized by scanning electron microscopy (SEM) and differential scanning calorimetry (DSC). For analysis with SEM, parts were cut from the membranes (before drying), fractured in liquid nitrogen and dried in vacuo. Cross sections, top surfaces and bottom surfaces were transferred to a Balzers Union sputter unit, provided with a gold layer and examined using a Jeol 35CF scanning electron microscope. For DSC analysis samples of $5 \mathrm{mg}$ were cut from the membranes and heated with a scan rate of $10^{\circ} \mathrm{C} / \mathrm{min}$ from 30 to $220^{\circ} \mathrm{C}$ in sealed aluminum sample pans.

\subsection{Phase diagrams}

Phase diagrams were constructed for the systems PLLA-chloroform-methanol and PDLLA-chloroform-methanol. Details will be reported in a subsequent paper. Cloud point curves were constructed by slowly cooling $\left(0.1^{\circ} \mathrm{C} / \mathrm{min}\right)$ the solutions from the homogeneous state till the solutions became turbid (detection by laser light transmission). The cloud point curve at $25^{\circ} \mathrm{C}$ was obtained by interpolation. Estimations for the glass transitions were obtained from data of Triolo on the depression of the glass transition of PLLA and PDLLA by dichloromethane [19].

\section{Results and discussion}

\subsection{Characterization of the polymers}

The intrinsic viscosity, the optical rotation and the thermal properties of the polymers synthesized are presented in Table 1.

The optical rotation values obtained for PLLA indicates that this polymer can be regarded as optically pure [18]. PDLLA was optically inactive as should be expected for a racemic copolymer. The optical rotations of PLA95 and PLA80 were 90 and $60 \%$ of the value attained for PLLA, respectively, and are in accordance with the excess enantiomer present. Melting transitions were only obtained for PLLA and PLA95. The melting temperatures of the different fractions of PLLA decreased slightly with decreasing molecular weight. The rate of crystallization, the melting temperature and the heat of fusion of PLA95 were much lower than for PLLA. PLLA already crystallized during cooling from the melt. PLA95 only crystallized after aging. PDLLA and PLA80 did not crystallize from the melt under the conditions of the experiment. When aged for 1 week at room temperature the heat of fusion of PLA95 increased to $15 \mathrm{~J} / \mathrm{g}$. After aging the heat of fusion of the PLLA samples increased to $40 \mathrm{~J} / \mathrm{g}$ for the high molecular weight samples and to $50 \mathrm{~J} / \mathrm{g}$ for the lowest molecular weight sample. The heat of fusion for $100 \%$ crystalline PLLA is reported to be $140 \mathrm{~J} / \mathrm{g}$ [20]. Hence PLA95 and PLLA can be regarded as moderately crystalline polymers. The glass transitions of the polymers range from 55 (PDLLA) to $60^{\circ} \mathrm{C}$ (PLLA).

\subsection{Phase diagrams}

In Fig. 1 the phase diagrams for the combinations PLLA-chloroform-methanol and PDLLA-chloroform-methanol are shown. The initial compositions of the casting solutions used for the preparation of membranes are indicated with an arrow. A considerable amount of methanol is required to induce demixing of the solutions. At low polymer concentrations the cloud point curves of PLLA and PDLLA are very close to each other. The cloud point curve of PDLLA can only 
Table 1

Poperties of the polylactide stereoisomers after cooling from the melt

\begin{tabular}{llclc}
\hline Polymer & Intrinsic viscosity $(\mathrm{dl} / \mathrm{g})$ & Optical rotation $[\alpha]_{\mathrm{D}}^{25}$ & Melting point $\left({ }^{\circ} \mathrm{C}\right)$ & Heat of fusion $(\mathrm{J} / \mathrm{g})$ \\
\hline PLA 100(PLLA) & $0.7-4.7^{\mathrm{a}}$ & -158 & $189^{\mathrm{a}, \mathrm{b}}$ & 35 \\
PLA95 & 5.1 & -143 & 163 & 3 \\
PLA80 & 4.5 & -96 & & \\
PLA50(PDLLA) & 5.5 & 0 & & \\
\hline
\end{tabular}

aSeveral samples with varying intrinsic viscosities were used.

${ }^{\mathrm{t}} \mathrm{The}$ value for the highest intrinsic viscosity is indicated. Lower molecular weight samples have slightly lower melting points.

be attributed to liquid-liquid demixing processes. This was confirmed by the fact that during prolonged aging of the phase separated solutions two completely separated transparent layers were obtained.

Homogeneous solutions of PLLA in chloroform can be prepared with polymer concentrations upto $30 \% \mathrm{w} /$ w. At higher polymer concentrations the polymer crystallizes from solution. The influence of crystallization processes also becomes clear from the cloud point curve for PLLA. The cloud point curve slowly bends towards lower methanol concentrations at increasing PLLA concentrations. At low PLLA concentrations the cloud point curve is due to liquid-liquid demixing processes. At high polymer concentrations the cloud point curve is due to solid-liquid demixing. For PLLA the

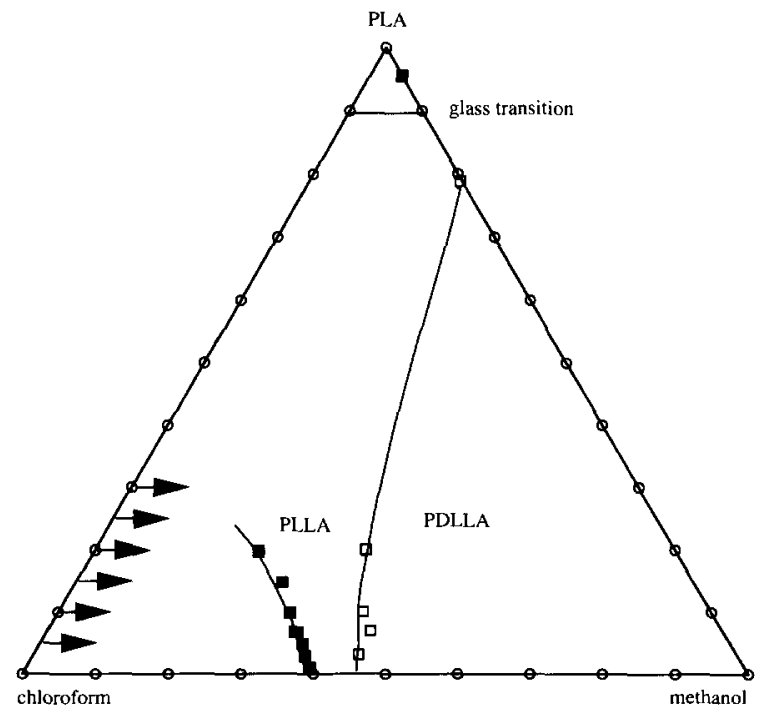

Fig. 1. Cloud point curves and glass transitions for the systems PLLA-chloroform-methanol and PDLLA-chloroform-methanol. Temperature, $25^{\circ} \mathrm{C}$. Symbols at the polymer-nonsolvent axis are swelling values of the polymer in pure nonsolvent. The arrows represent the compositions of the casting solutions used. cloud point curve is not likely to represent an equilibrium transition because crystallization phenomena are generally cooling rate dependent.

The glass transition at $25^{\circ} \mathrm{C}$ is located at approximately $10 \%$ solvent for both PLLA and PDLLA. The swelling value of PDLLA in methanol is ca. $22 \% \mathrm{w} /$ w. Due to its high crystallinity the swelling value of PLLA is much lower $(7 \% \mathrm{w} / \mathrm{w})$. A more detailed discussion about the phase diagrams will be reported in a forthcoming paper.

\subsection{Membranes prepared from PDLLA}

The precipitation of solutions of PDLLA in chloroform in methanol was rather peculiar. The solutions became turbid within $30-40 \mathrm{~s}$. However after prolonged equilibration of the membranes in the nonsolvent the turbidity gradually disappeared. Fig. 2 a shows the morphology of a membrane dried after $4.5 \mathrm{~h}$ of incubation in methanol (polymer concentration $5 \% \mathrm{w} /$ $w$ ). The cross section of the membrane consists of a very open porous structure without a gradient in pore size over the membrane thickness. When the membranes are kept in methanol for 1 day or longer the cross sections of the membranes do not contain pores anymore (Fig. 2b).

For the polymer-solvent-nonsolvent combination investigated the only mechanism capable of stabilizing the demixed solution is vitrification of the polymer rich phase. The glass transition of PDLLA is located at $55^{\circ} \mathrm{C}$. From the phase diagram (Fig. 1) it becomes clear that $10 \%$ solvent or nonsolvent is already sufficient to decrease the glass transition of the polymer from $55^{\circ} \mathrm{C}$ to room temperature. The swelling of PDLLA in methanol at $25^{\circ} \mathrm{C}$ amounts to $22 \% \mathrm{w} / \mathrm{w}$ methanol. Apparently the chains are sufficiently mobile to allow significant coarsening of the demixed structure. Phase 

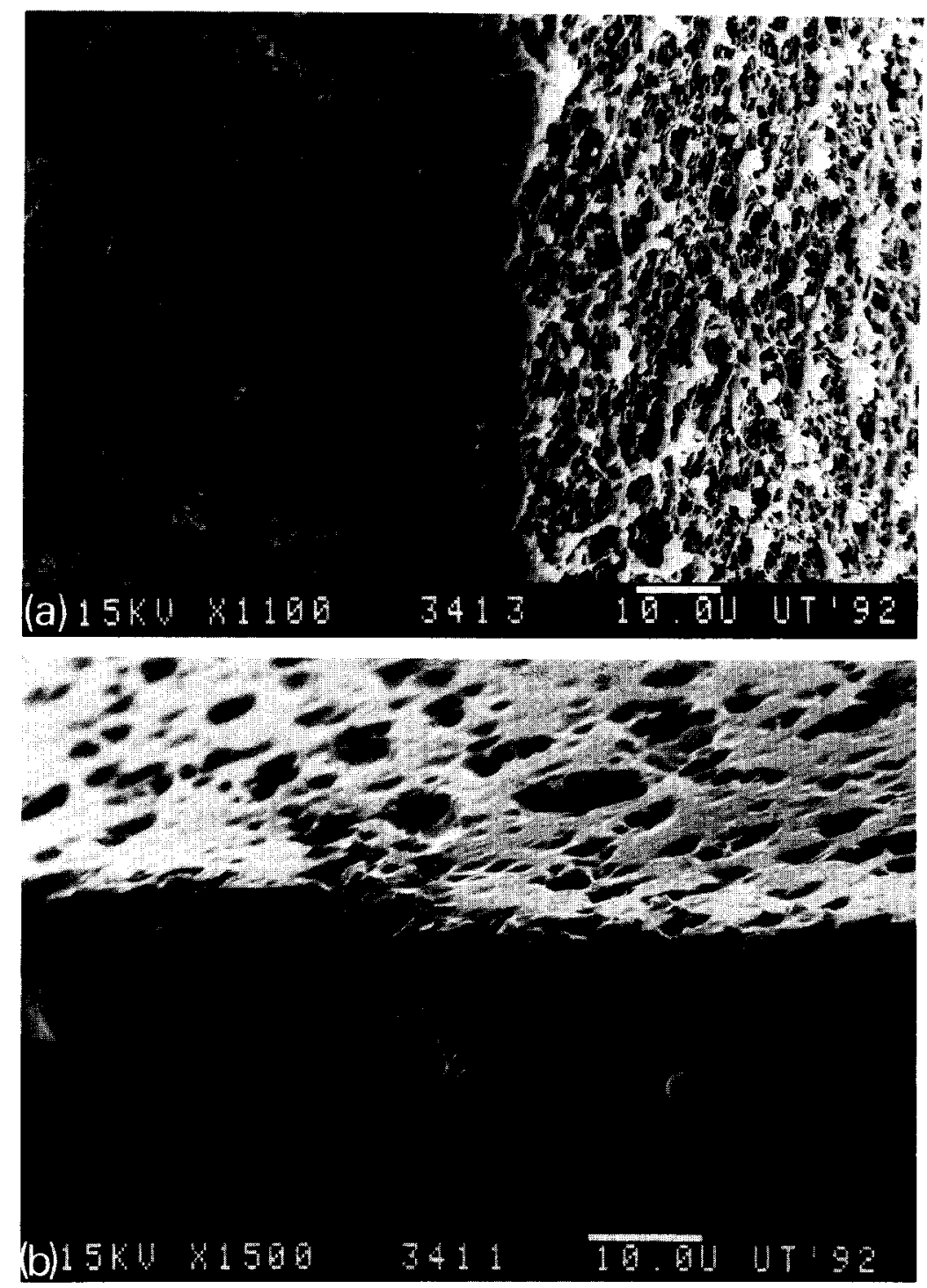

Fig. 2. Cross sections of membranes prepared from a $5 \% \mathrm{w} / \mathrm{w}$ solution of PDLLA ( $\eta]=5.1 \mathrm{dl} / \mathrm{g})$. (a) edge view of membrane, $4.5 \mathrm{~h}$ after immersion in methanol. (b) The same membrane after 1 day of incubation in methanol.

separation can proceed until two completely separated layers are obtained.

\subsection{Membranes prepared from PLA95 and PLA80}

These stereoisomers have a glass transition at almost the same temperature as PDLLA. Because the structure of the amorphous PDLLA formed initially after immersion is not stable in the nonsolvent, the only other transition that is potentially capable of solidifying the demixed phases of polylactides is solid-liquid demixing. From Table 1 it can be concluded that PLA 80 does not easily crystallize from the melt. Therefore, it is not surprising that also no stable porous membranes could be obtained with PLA80. The precipitation behaviour was the same as for PDLLA. The membranes prepared with PLA80 display a small but significant degree of crystallinity (see Table 2 ). The melting point of the 
Table 2

Melting points and heats of fusion of the membranes

\begin{tabular}{llll}
\hline Polymer & $|\eta|(\mathrm{d} l / \mathrm{g})$ & $\begin{array}{l}\text { Melting temperature } \\
\left({ }^{\circ} \mathrm{C}\right)\end{array}$ & $\begin{array}{l}\text { Heat of fusion } \\
(\mathrm{J} / \mathrm{g})\end{array}$ \\
\hline PLLA & 0.7 & 175 & 50 \\
PLLA & 2 & 180 & 45 \\
PLLA & 3 & 190 & 40 \\
PLLA & 4.7 & 185 & $40(5)^{\mathrm{a}}$ \\
PLA95 & 5.1 & 161 & 13 \\
PLA80 & 4.5 & 97 & 1 \\
PDLLA & 5.1 & & \\
\hline
\end{tabular}

"Number between brackets indicates the heat of recrystallization during heating.

crystals is located at temperatures only slightly higher than the glass transition temperature of PLA80. Generally the rate of crystallization decreases strongly in the vicinity of the glass transition [21]. Most likely the crystallization of PLA 80 in the membranes is enhanced by the lowering of the glass transition of the polymer by the methanol. Since only dense membranes were obtained the rate of crystallization must have been too slow to prevent complete demixing of the solution.

PLA95 crystallizes sufficiently rapid to stabilize the membrane structure (Fig. 3). The crystallinity of these membranes is also significantly higher than the samples that were cooled from the melt (see Tables 1 and 2). The membranes have a very regular porous structure and are moderately crystalline. The circular shape of the pores indicates that phase separation has occurred initially by liquid-liquid demixing by nucleation and growth of a polymer poor phase. Solid-liquid demixing in the polymer rich phase must have been rapid enough to prevent a complete collapse of the demixed structure. Still the membranes obtained were shrunk laterally to a large extent and had poor mechanical properties.

\subsection{Membranes prepared from PLLA}

Poly-L-lactide is the most rapidly crystallizing polymer used in this study. In most of the experiments performed in this study PLLA was used with an intrinsic viscosity of $0.7 \mathrm{dl} / \mathrm{g}$. Using this molecular weight fraction membranes could be prepared from solutions containing 15,20, 25 and $30 \% \mathrm{w} / \mathrm{w}$ polymer (range due to viscosity requirements for casting). To enable the preparation of membranes with lower polymer concentrations also higher molecular weight fractions were used. PLLA with an intrinsic viscosity of $2 \mathrm{dl} / \mathrm{g}$ could be used for the preparation of solutions with concentrations of 10,15 and $20 \% \mathrm{w} / \mathrm{w}$. PLLA fractions with intrinsic viscosities of 3 and $4.7 \mathrm{dl} / \mathrm{g}$ were used for the preparation of 5 and $10 \% \mathrm{w} / \mathrm{w}$ solutions.

All casting solutions yielded stable membranes. The membranes prepared with PLLA fractions with low intrinsic viscosities $(0.7-2 \mathrm{dl} / \mathrm{g})$ were very fragile and

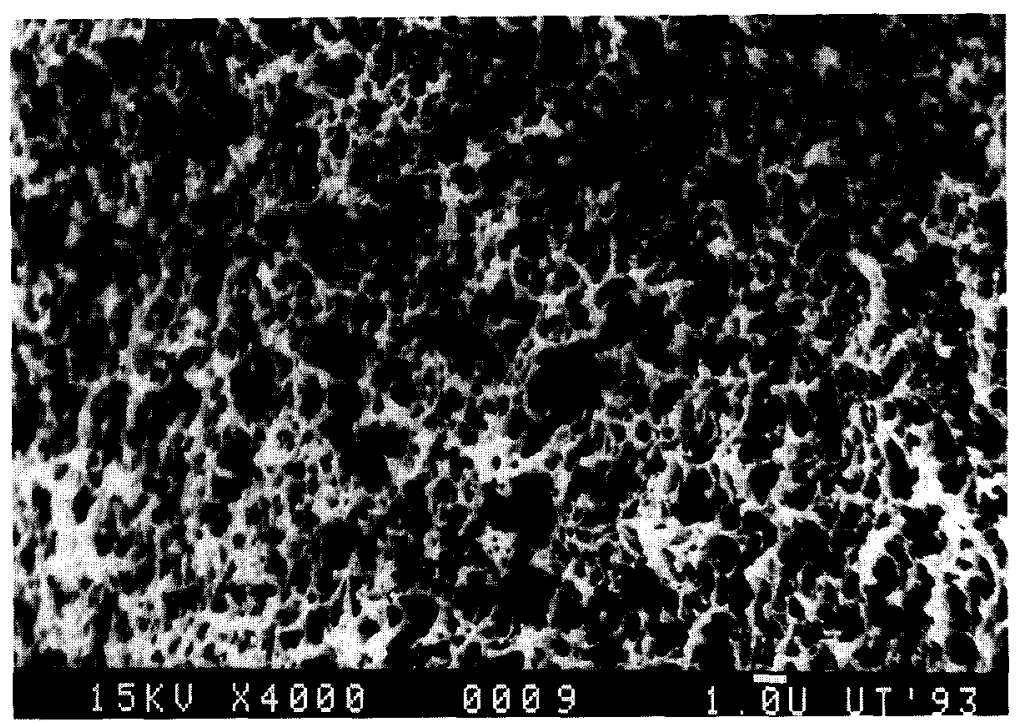

Fig. 3. Cross section of a membrane prepared from a $5 \% \mathrm{w} / \mathrm{w}$ solution of PLA95 in chloroform. 


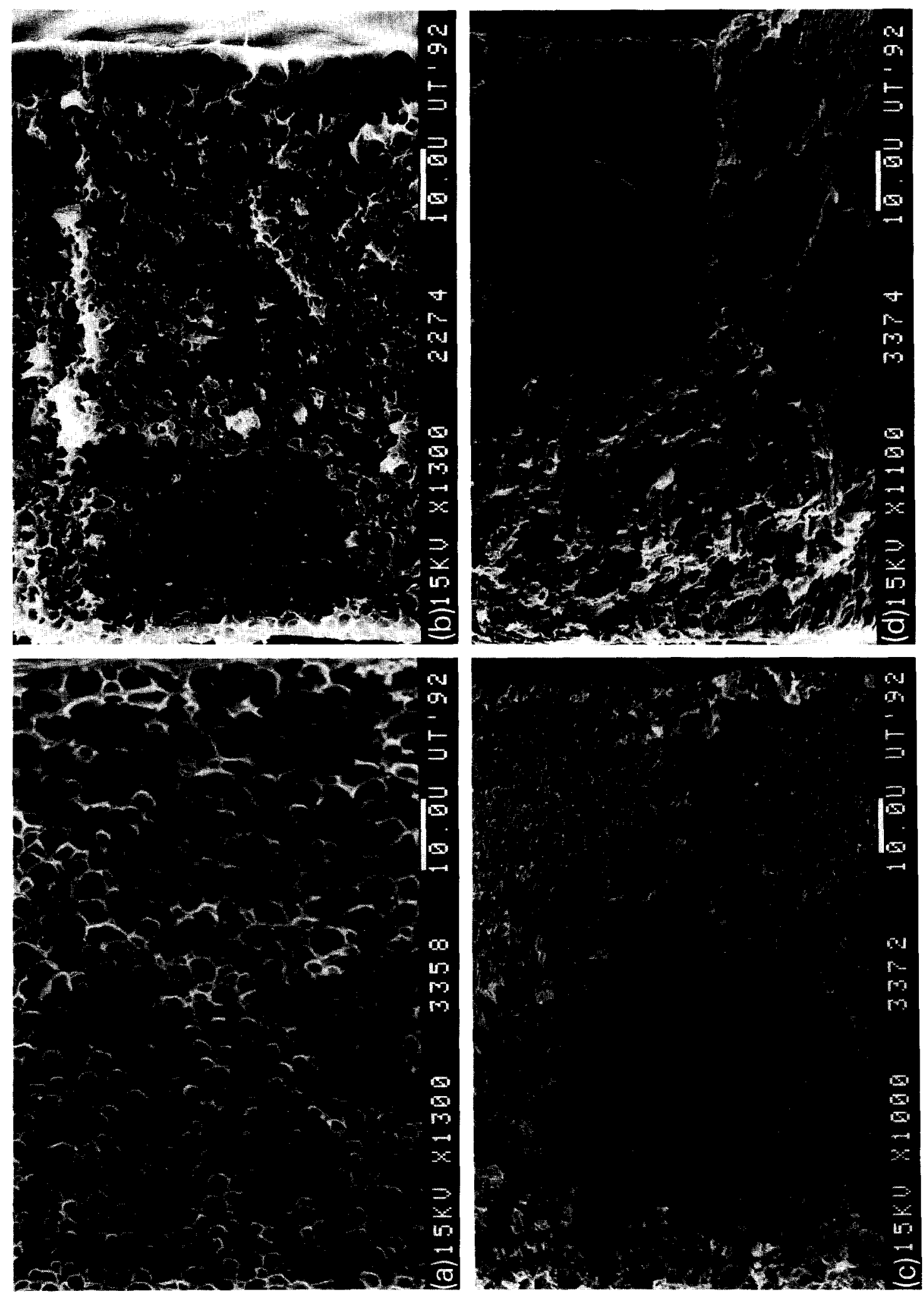

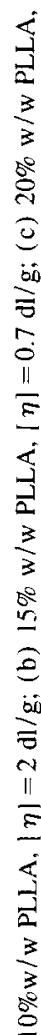

ล

$\div$.

惫

茞

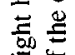
놀 ล

8

呅

훙훙

이

宽

है

$<\overrightarrow{0}$

3 $\pm 3$ 它 ๘ ํำ 


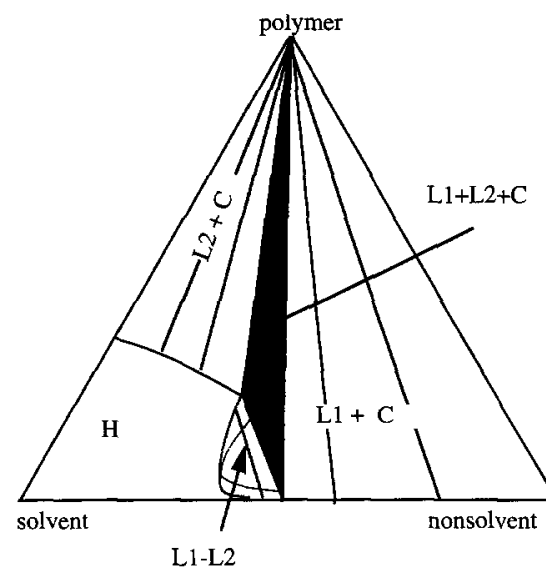

(A)

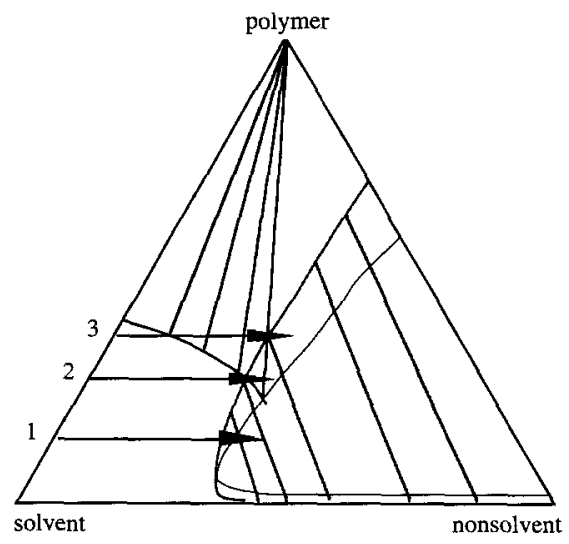

(B)

Fig. 5. (A) Equilibrium phase diagram for a polymer-solvent-nonsolvent system for which both liquid-liquid demixing and solid-liquid demixing are thermodynamically important. L1, dilute polymer solutions; L2, concentrated polymer solution; C, crystalline phase; $\mathrm{H}$, homogeneous solution. (B) Non-equilibrium phase diagram. The entire liquid-liquid miscibility gap is indicated including the part that does not have significance under equilibrium conditions. Also the crystallization transition is extended partially into the miscibility gap. For numbers and arrows, see text. Notice that in (A) the equilibrium melting transition is indicated and in (B) a corresponding (arbitrary) crystallization transition.

not suitable for any application. The strength of the membranes however strongly increased when PLLA fractions with higher intrinsic viscosities were used. The lateral shrinkage of all membranes obtained with PLLA was much smaller than the shrinkage observed with membranes obtained with PLA95.

The morphologies of the membranes depended strongly on the polymer concentration of the casting solution. No large differences in membrane structure were observed between the membranes prepared with the same polymer concentration of the casting solution but with different molecular weight fractions. The cross sections of the membranes prepared from solutions containing up to $15 \% \mathrm{w} / \mathrm{w}$ polymer contain circular pores and have a high porosity (Figs. $4 \mathrm{a}$ and $4 \mathrm{~b}$ ). The pores in the cross sections of the membranes are due to nucleation and growth of a polymer poor phase. Most likely after liquid-liquid demixing the solid-liquid demixing process takes care of the fixation of the structure [1]. The suggested mechanism of liquid-liquid demixing followed by solid-liquid demixing is supported by the rather long precipitation times. The delay times for demixing were comparable with the delay times for the precipitation of the copolymers $( \pm 40 \mathrm{~s})$.

The cross sections of the membranes prepared with a polymer concentration of $20 \% \mathrm{w} / \mathrm{w}$ consist of circular pores (Fig. 4c). However superimposed on the cellular morphology the contours of circular sections can be recognized. In the same membranes also sections are found with a dense core surrounded by porous fingers radiating to the outside (Fig. 4d). It is possible that some distance behind the fully porous cross sections also dense centers are present. The elongated shapes of the pores at the borders of the different sections suggest a spherical geometry. These large spherical structures were also frequently obtained by Lloyd and co-workers during thermally induced phase separation and are most likely spherulites [6]. The formation of spherulites from thermally quenched concentrated solutions of PLLA in acetonitrile was demonstrated earlier by Tsuji et al. [22].

The occurrence of both demixing processes can be explained by schematic phase diagrams. In Fig. 5A an equilibrium phase diagram is constructed for a polymer-solvent-nonsolvent system. For the indicated system solid-liquid equilibria and liquid-liquid equilibria are thermodynamically favoured in certain regions of the phase diagram. The three binary phase equilibria are separated from each other by a three phase equilibrium. Because crystallization of a polymer from solution is a non-equilibrium phenomenon, non-equilibrium phase diagrams are needed to explain the structure formation. In Fig. 5B a corresponding non-equilibrium phase diagram is presented. Both the liquid-liquid mis- 

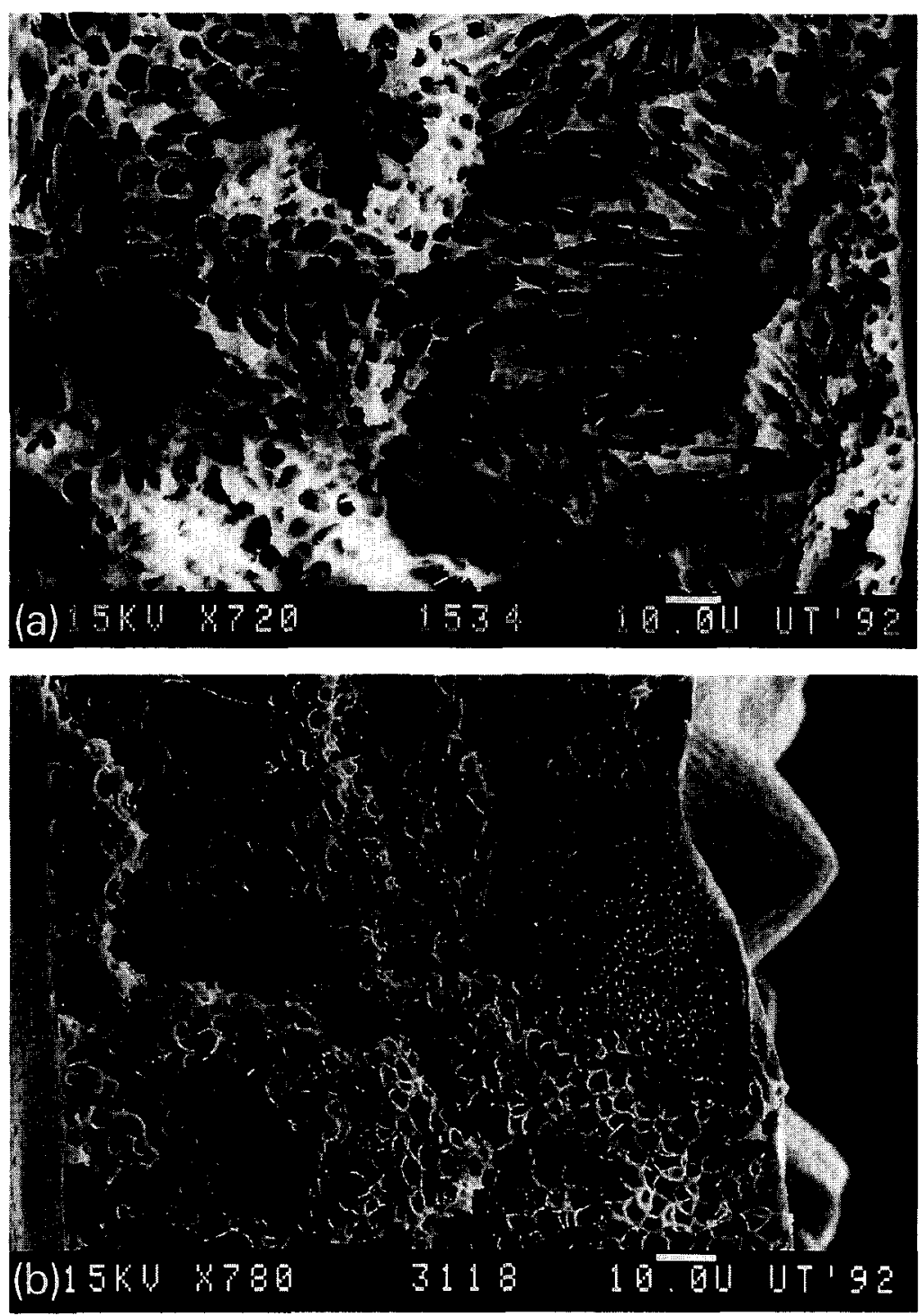

Fig. 6. Cross section of membranes of PLLA (top surfaces at the right hand side). (a) casting solution: 20\% w/w PLLA-64\% w/w chloroform$16 \% \mathrm{w} / \mathrm{w}$ methanol; $|\eta|=0.7 \mathrm{dl} / \mathrm{g}$. (b) casting solution: $20 \% \mathrm{w} / \mathrm{w}$ PLLA-80\% w/w chloroform; $|\eta|=2 \mathrm{dl} / \mathrm{g}$.

cibility gap and the crystallization transition are shown independently. Also the tielines for the transitions are indicated. The cloud point curve of the indicated kinetic phase diagram (Fig. 5B) resembles the cloud point curve of the phase diagram found experimentally (Fig. 1). Arrow 1 represents schematically the path followed by the polymer solutions with low polymer concentrations. First the liquid-liquid miscibility gap is entered and the polymer rich phase crystallizes subsequently.
For path 2 crystallization and nucleation and growth can occur at the same time. For binary systems this kind of demixing process has been studied in detail by $\mathrm{Li}$ et al. [23]. They studied the demixing of a semicrystalline polymer (poly- $\varepsilon$-caprolactone) and an amorphous polymer (polystyrene) using optical microscopy. In some areas of the temperature-concentration phase diagram of the blend the poly- $\varepsilon$-caprolactone rich phase started to crystallize slowly after the liquid-liquid demixing 

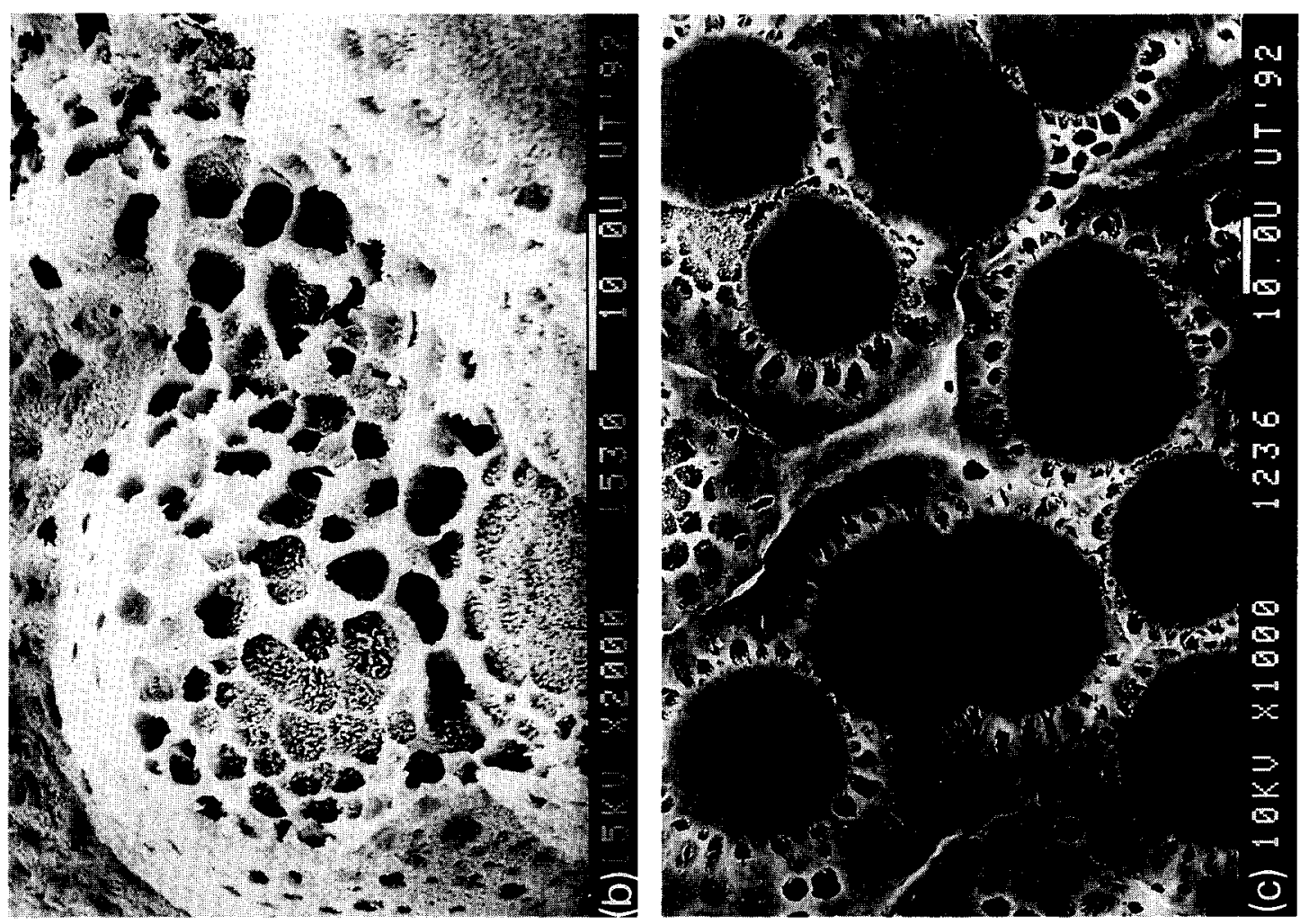

$\stackrel{5}{\stackrel{0}{0}}$
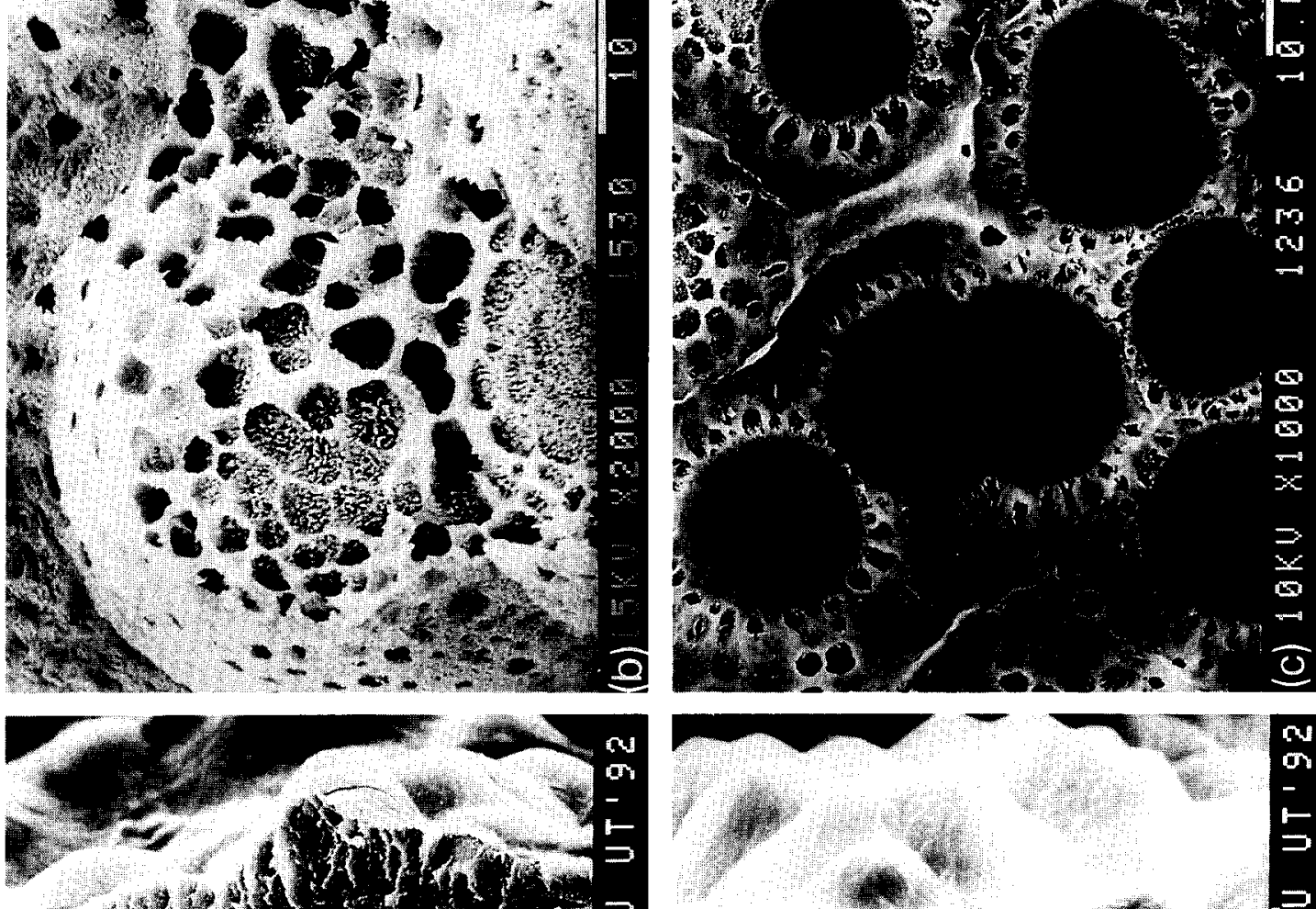

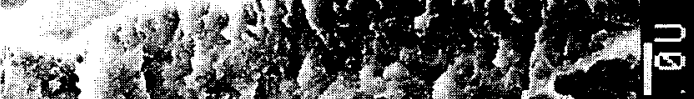

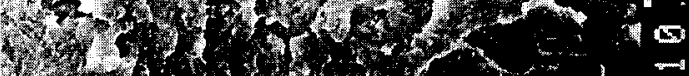
3.
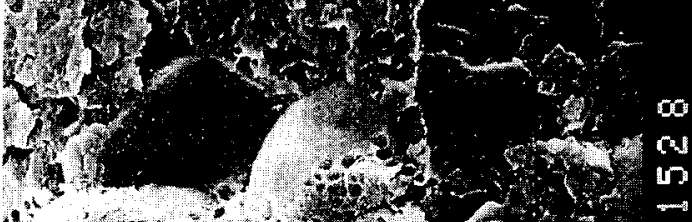

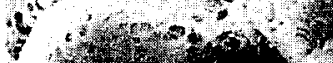
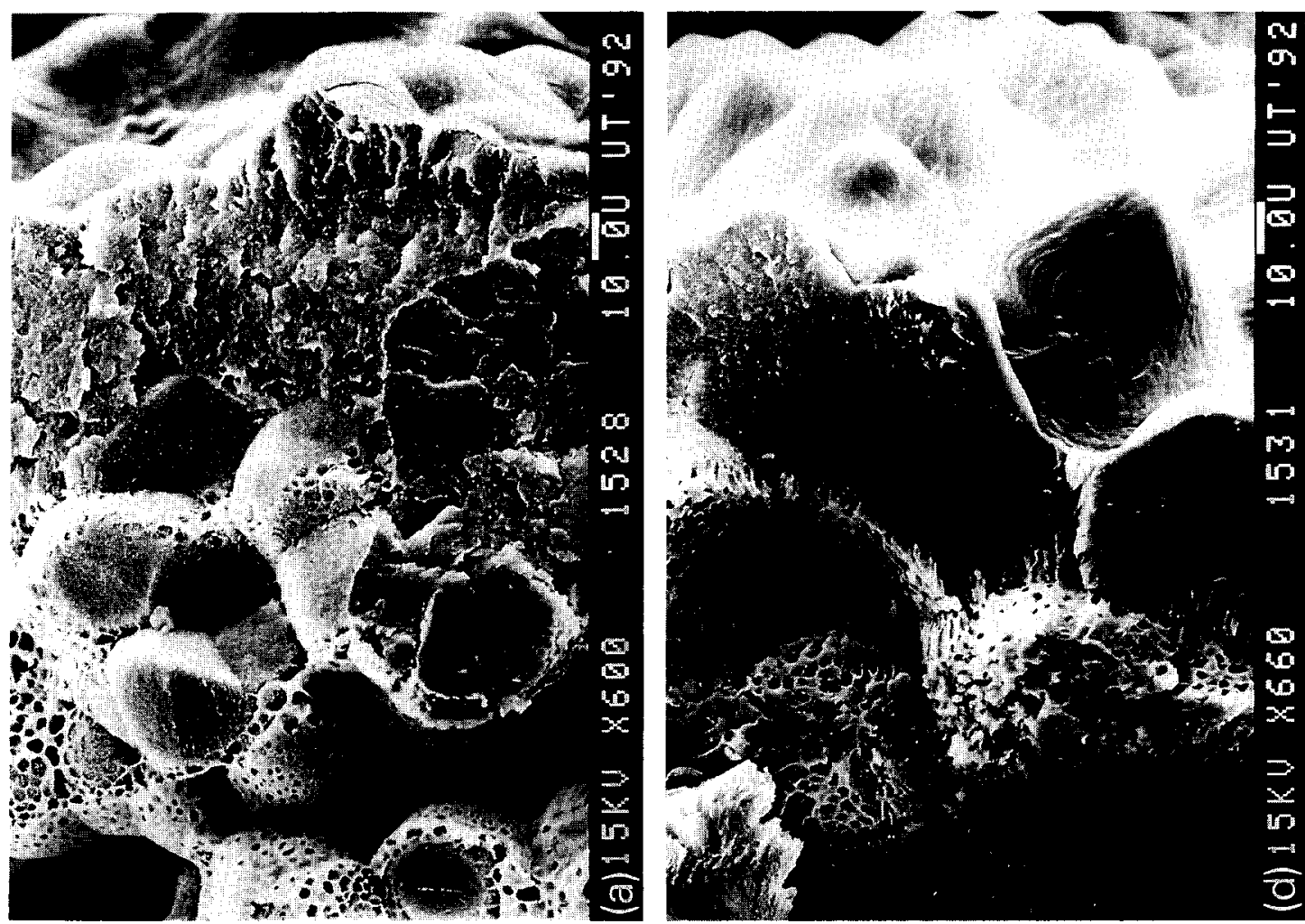

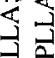

3

3

i

تี

吾

on

范

ส

恋

吾

记

莺

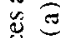

造

䟢

을

F

○ं

E

$\leqslant \Xi$

긍

$\stackrel{0}{E}$

豆

5

咅

ํํㄹ

옹

嵩

导

⿷

홀

일

น

导

交合

본

ำ 

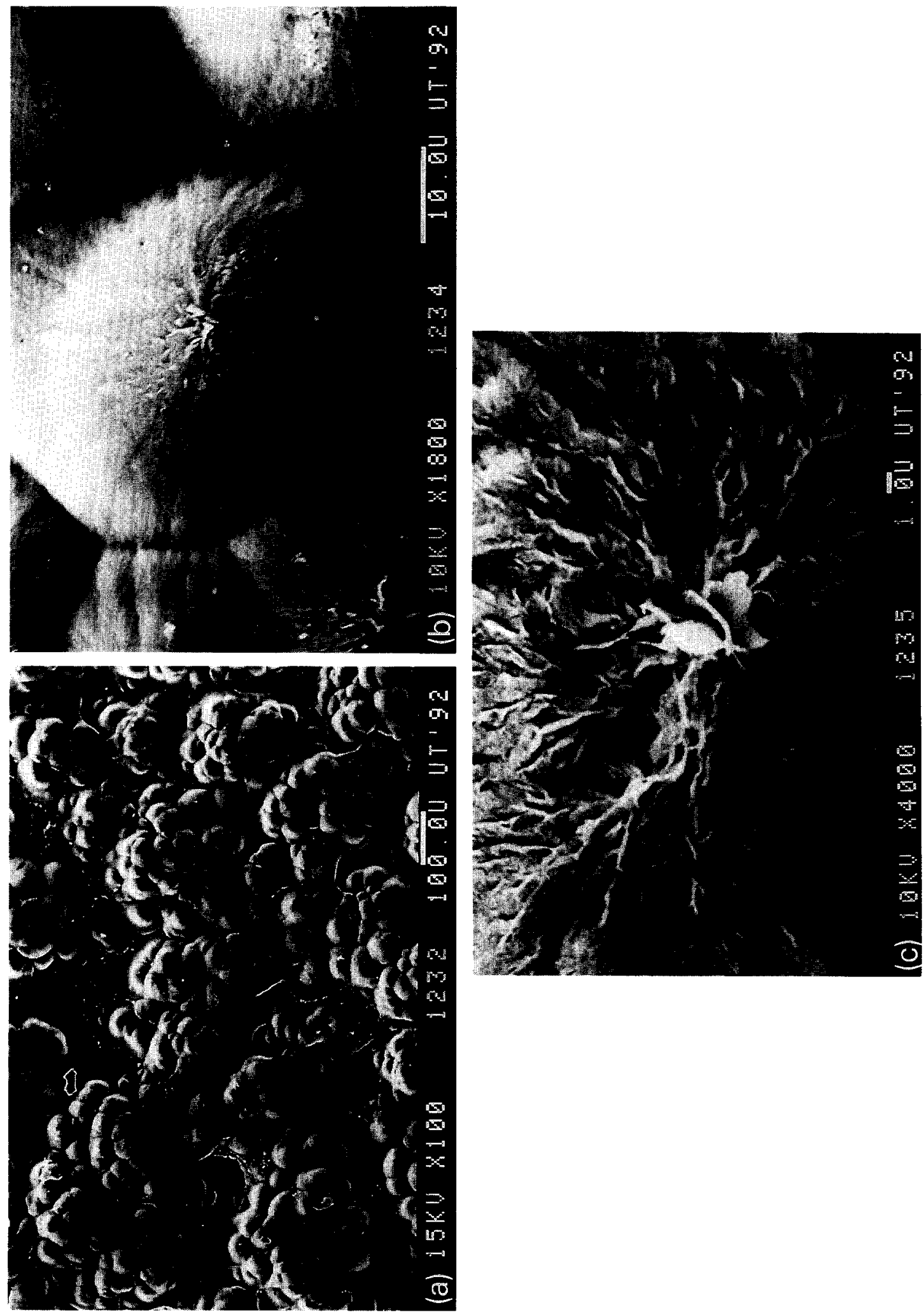

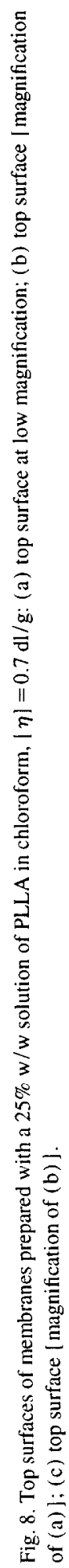


process had generated droplets of polystyrene. The spherulites were shown to engulf the polystyrene droplets during growth. Coalescence of the droplets can lead to the porous fingers found in our membranes. Another possibility is that the droplets grow simultaneously with the spherulite. It was observed that when some chloroform in the casting solution was replaced by methanol (chloroform-methanol ratio 80:20\% w/w) the size of the fingers increased dramatically at the expense of the more dense centre of the spherulite (Fig. $6 a)$. Apparently liquid-liquid demixing interferes in an earlier stage of the spherulite growth.

The membranes prepared from chloroform solutions with a polymer concentration of $20 \% \mathrm{w} / \mathrm{w}$ but with an intrinsic viscosity of $2 \mathrm{dl} / \mathrm{g}$ also contain dense areas embedded in a porous matrix (Fig. 6b). However no porous fingers can be observed in the cross section. Subtle differences in rates of phase separation or coagulation paths may be the reason for this difference.

More concentrated solutions $(25 \% \mathrm{w} / \mathrm{w},[\eta]=0.7$ $\mathrm{dl} / \mathrm{g}$ ) turn turbid almost instantaneously after immersion. The membranes obtained also contain spherulites in the lower part of the cross sections (Figs. 7a and $7 \mathrm{~b})$. The number of spherulites per unit volume is higher than in the case of the membranes obtained from PLLA solutions of $20 \% \mathrm{w} / \mathrm{w}$ and $[\eta]=0.7 \mathrm{dl} / \mathrm{g}$. The spherulites are smaller and have a dense core. However signs of structures due to liquid-liquid demixing can still be recognized. A porous shell covers the spherulites. At the bottom surface the contours of the spherulites surrounded with smaller pores can also be recognized (Fig. 7c). Spherulites surrounded with pores were also frequently observed by Li et al. [23], Tanaka and Nishi [24] and Shibanov et al. [25] during the phase separation of mixtures of crystalline and amorphous polymers. The formation process is closely related to the formation of the fingered spherulites. In these cases solid-liquid demixing induces liquid-liquid demixing just in front of the growing spherulite (path 3 in Fig. 5). There are no indications for liquid-liquid phase separation within the spherulite. The part of the cross section close to the top surface is rather dense. Pores can only be distinguished at high magnifications $(10000-15000 \times)$. It is expected that this dense part consists of spherulites fused to such an extent that no distinction of the spherulites is possible anymore. When the polymer concentration of the casting solution is increased to $30 \% \mathrm{w} / \mathrm{w}$ the spherulites in the lower part of the cross section are more fused together (Fig. 7d). These spherulites are somewhat flattened and contain large pores. The general features are similar to the membranes obtained with solutions of 20 and $25 \%$ w/ w polymer.

Spherulites can be recognized in the top surfaces of all membranes prepared from casting solutions with polymer concentrations higher than $10 \% \mathrm{w} / \mathrm{w}$. The higher the polymer concentration of the casting solution the more the spherulites stick out of the top surfaces of the membranes.

In Figs. 8a-8c micrographs of the spherulites that are present in the top surface area of the membranes prepared with a polymer concentration of $25 \% \mathrm{w} / \mathrm{w}$ are presented. The fine texture of lamellar stacks can easily be recognized at the top surface of the spherulites. The spherulites at the top surface are very prominent. The distinct presence of spherulites in the top surface possibly results from an asymmetric distribution of the polymer concentration over the membrane thickness. The exchange of solvent for nonsolvent induces a very rapid increase of the polymer concentration at the interface directly after immersion [2628]. A second possible reason for elevated polymer concentrations at the interface is evaporation of the solvent during the time between casting and immersion. However when membranes were immersed in the nonsolvent without evaporation step the structure of the membranes prepared was very similar.

\section{Conclusions}

The morphology of the polylactide membranes is the result of a complex interplay between solid-liquid demixing and liquid-liquid demixing. The glass transition did not play an important role in the structure formation because of the high swelling of the polymers in the nonsolvent. Stable membrane structures could only be obtained with the rapidly crystallizing polymers, PLA95 and PLLA. The polymer concentration of the casting solution determined whether the structure formation occurred by liquid-liquid demixing followed by solid-liquid demixing or by solid-liquid demixing followed by liquid-liquid demixing. The morphologies of the membranes were in line with the features of the experimentally determined phase diagrams. 


\section{References}

[1] M. Mulder, Basic Principles of Membrane Technology, Kluwer Academic Publishers, Dordrecht, 1992.

12] (a) J.-M. Guenet, Thermoreversible Gelation of Polymers and Biopolymers, Academic Press, London, 1992; (b) M.J. Miles, Gelation, in D.C. Bassett (Ed.), Developments in Crystalline Polymers-2; Elsevier Applied Science, New York, 1988, pp. 233-295.

[3] P. Vandeweerdt, H. Berghmans and Y. Tervoort, Temperatureconcentration behavior of solutions of polydisperse, atactic poly (methylmethacrylate) and its influence on the formation of amorphous, microporous membranes, Macromolecules, 24 (1991) 3547.

[4] R.M. Hikmet, S. Callister and A. Keller, Thermoreversible gelation of atactic polystyrene: phase transformation and morphology, Polymer, 29 (1988) 1378.

[5] A. Prasad, H. Marand and L. Mandelkern, Supermolecular morphology of thermoreversible gels formed from homogeneous and heterogeneous solutions, J. Polym. Sci., 32 (1993) 1819.

[6] (a) D.R. Lloyd, K.E. Kinzer and H.S. Tseng, Microporous membrane formation via thermally induced phase separation. I. Solid-liquid phase separation, J. Membrane Sci., 52 (1990) 239; (b) D.R. Lloyd, S.S. Kim and K.E. Kinzer, Microporous membrane formation via thermally induced phase separation. II. Liquid-liquid phase separation, ibid., 64 (1991) 1; (c) S.S. Kim and D.R. Lloyd, Microporous membrane formation via thermally induced phase separation. III. Effect of thermodynamic interactions on the structure of isotactic propylene membranes, ibid., 64 (1991) 13.

17| P. Radovanovic, S.W. Thiel and S.-T. Hwang, Formation of asymmetric polysulfone membranes by immersion precipitation. Part II. The effect of casting solution and gelation bath compositions on membrane structure and skin formation, J. Membrane Sci., 65 (1992) 230.

|8| G.E. Gaides and A.J. McHugh, Gelation in an amorphous polymer: a discussion of its relation to membrane formation, Polymer, 30 (1989) 2118.

[9] W.R. Burghardt, L. Yilmaz and A.J. McHugh, Glass transition, crystallization and thermoreversible gelation in ternary PPO solutions: relationship to asymmetric membrane formation, Polymer, 28 (1987) 2085.

$110 \mid$ J.G. Wijmans, J.P.B. Baay and C.A. Smolders, The mechanism of formation of microporous or skinned membranes produced by immersion precipitation, J. Membrane Sci., 14 (1983) 263.

111 A.J. Reuvers and C.A. Smolders, Formation of membranes by means of immersion precipitation. Part II. The mechanism of formation of membranes prepared from the system cellulose acetate-acetone-water, J. Membrane Sci., 29 (1987) 67.

| 12 | A.M.W. Bulte, B. Folkers, M.H.V. Mulder and C.A. Smolders, Membranes of semicrystalline aliphatic polyamide nylon-4,6: formation by diffusion-induced phase separation, J. Appl. Polym. Sci., 50 (1993) 13.
[13] M.J.D. Eenink, J. Feijen, J. Olijslager, J.H.M. Albers, J.C Rieke and P.J.J. Greidanus, Biodegradable hollow fibres for the controlled release of hormones, J. Controlled Release, 6 (1987) 225.

[14] P. Van de Witte, H. Esselbrugge, A.M.P. Peters, P.J. Dijkstra, J. Feijen, R.J.J. Groenewegen, J. Smid, J. Olijslager, J.M. Schakenraad, M.J.D. Eenink and A.P. Sam, Formation of porous membranes for drug delivery systems, J. Controlled Release, 24 (1993) 61.

[15] H. Esselbrugge, Biodegradable Hollow Fibres for Controlled Drug Delivery, Ph.D. Thesis, University of Twente (The Netherlands), 1992.

[16] F. Chabot, M. Vert, S. Chapelle and P. Granger, Configurational structures of lactic acid stereocopolymers, Polymer, 24 (1983) 53.

[17] P.J.A. In't Veld, P.J. Dijkstra, J. Van Lochem and J. Feijen, Synthesis of alternating polydepsipeptides by ring-opening polymerization of morpholine-2,5-dione, Makromol. Chem. 191 (1990) 1813.

[18] H.R. Kricheldorf and A. Serra, Polylactones 6. Influence of various metal salts on the optical purity of poly (L-lactide), Polym. Bull., 14 (1985) 497.

[19] P.M. Triolo, The Release of Bovine Serum Albumin from Biodegradadable Poly-L-lactic acid Matrices, Ph.D. Thesis, University of Utah, 1987.

[20] G.L. Loomis, J.R. Murdoch and K.H. Gardner, Polylactide stereocomplexes, Polym. Prepr., 31(2) (1990) 55

[21] (a) A. Marega, A. Marigo, V. Di Noto, R. Zannetti, A. Martorana and G. Paganetto, Structure and crystallization kinetics of poly (L-lactic acid), Makromol. Chem., 193 (1992) 1599; (b) R. Vasanthakumari, A.J. Pennings, Crystallization kinetics of poly (L-lactic acid), Polymer, 24 (1983) 175.

[22] H. Tsuji, S.-H. Hyon and Y. Ikada, Stereocomplex formation between enantiomeric poly (lactic acid)s. 5. Calorimetric and morphological studies on the stereocomplex formed in acetonitrile solution, Macromolecules, 25 (1992) 2940.

[23] (a) Y. Li, M. Stein and B.-J. Jungnickel, Competition between crystallization and phase separation in polymer blends. I. Diffusion controlled supermolecular structures and phase morphologies in poly ( $\varepsilon$-caprolactone $) /$ polystyrene blends, Colloid Polym. Sci., 269 (1991) 772; (b) Y. Li and B.-J. Jungnickel, The competition between crystallization and phase separation in polymer blends: 2 . Small angle $\mathrm{X}$-ray scattering studies on the crystalline morphology of poly ( $\varepsilon$-caprolactone) in its blends with polystyrene, Polymer, 34 (1993) 9.

[24] (a) H. Tanaka and T. Nishi, New types of phase separation behaviour during the crystallization process in polymer blends with phase diagram, Phys. Rev. Lett., 55 (1985) 1102; (b) H. Tanaka and T. Nishi, Local phase separation at the growth front of a polymer spherulite during crystallization and nonlinear spherulitic growth in a polymer mixture with a phase diagram, Phys. Rev. A, 39 (1989) 783.

[25] Y.D. Shibanov and Y.K. Godovsky, Interrelation of phase and relaxation behaviour in polymer blends and block copolymers with crystallizable components, Prog. Colloid Polym. Sci., 80 (1989) 110. 
|26| A.J. Reuvers, J.W.A. Van den Berg and C.A. Smolders, Formation of membranes by means of immersion precipitation. Part I. A model to describe mass transfer during immersion precipitation, J. Membrane Sci., 29 ( 1987) 45.
[27] P. Radovanovics, S.W. Thiel and S.-T. Hwang, Formation of asymmetric polysulfone membranes by immersion precipitation. Part I. Modelling mass transport during gelation, J. Membrane Sci., 65 (1992) 213.

[28] C.S. Tsay and A.J. McHugh, Mass transfer modeling of asymmetric membrane formation by phase inversion, J. Polym. Sci., Part B, 28 (1990) 1327. 